\title{
A CHARACTERISATION OF NILPOTENT BLOCKS
}

\author{
RADHA KESSAR, MARKUS LINCKELMANN, AND GABRIEL NAVARRO
}

(Communicated by Pham Huu Tiep)

\begin{abstract}
Let $B$ be a $p$-block of a finite group, and set $m=\sum \chi(1)^{2}$, the sum taken over all height zero characters of $B$. Motivated by a result of $M$. Isaacs characterising $p$-nilpotent finite groups in terms of character degrees, we show that $B$ is nilpotent if and only if the exact power of $p$ dividing $m$ is equal to the $p$-part of $|G: P|^{2}|P: R|$, where $P$ is a defect group of $B$ and where $R$ is the focal subgroup of $P$ with respect to a fusion system $\mathcal{F}$ of $B$ on $P$. The proof involves the hyperfocal subalgebra $D$ of a source algebra of $B$. We conjecture that all ordinary irreducible characters of $D$ have degree prime to $p$ if and only if the $\mathcal{F}$-hyperfocal subgroup of $P$ is abelian.
\end{abstract}

\section{INTRODUCTION}

Let $p$ be a prime number and let $(K, \mathcal{O}, k)$ be a $p$-modular system. We assume that $k$ is algebraically closed and that $K$ is a splitting field for all finite groups considered in the paper. Let $G$ be a finite group and $B$ a block algebra of $\mathcal{O} G$. Let $\mathcal{F}=\mathcal{F}_{\left(P, e_{P}\right)}(G, B)$ be the fusion system of $B$ with respect to a maximal $B$-Brauer pair $\left(P, e_{P}\right)$. The focal subgroup of $\mathcal{F}$ is the subgroup $\mathfrak{f o c}(\mathcal{F})$ of $P$ generated by the commutators $\left[\operatorname{Aut}_{\mathcal{F}}(Q), Q\right]$, where $Q$ runs over the subgroups of $P$ (see [1, I, Def. 7.1]). Let $\operatorname{Irr}(B)$ denote the set of $K$-valued irreducible characters of $B$ and let $\operatorname{Irr}_{0}(B)$ denote the subset of height zero characters. Let $i \in B^{P}$ be a source idempotent of $B$ and let $S$ be a Sylow $p$-subgroup of $G$ containing $P$.

Theorem 1.1. With the notation above, the following are equivalent.

(i) $\left|\sum_{\chi \in \operatorname{Irr}_{0}(B)} \chi(1)^{2}\right|_{p}=|S: P|^{2}|P: \mathfrak{f o c}(\mathcal{F})|$.

(ii) $\left|\sum_{\chi \in \operatorname{Irr}_{0}(B)} \chi(i)^{2}\right|_{p}=|P: \mathfrak{f o c}(\mathcal{F})|$.

(iii) $\left|\operatorname{Irr}_{0}(B)\right|=|P: \mathfrak{f o c}(\mathcal{F})|$.

(iv) $B$ is nilpotent.

Theorem 1.1 may be seen as a block-theoretic analogue of Isaacs' result 9 , Lemma 4] characterising $p$-nilpotent groups via character degrees. The fact that statement (iv) in this theorem implies any of (i), (ii), (iii) is an immediate consequence of Puig's structure theorem [16, Theorem 1.6] for source algebras of nilpotent blocks. It is known that in general the quantity on the right-hand side of (i) or (ii) always divides the quantity on the left-hand side of (i) or (ii), respectively. This is a consequence of the free action of $P / \mathfrak{f o c}(\mathcal{F})$ on the set of height zero characters via the $*$-construction (see [17, Introduction], [18]). The proof of Theorem 1.1

Received by the editors February 24, 2014 and, in revised form, July 2, 2014 and September 30, 2014 .

2010 Mathematics Subject Classification. Primary 20C20.

Key words and phrases. Nilpotent block, height zero, hyperfocal subalgebra. 
relies on the Clifford theoretic relationship between the representation theory of the source algebra $i B i$ and its hyperfocal subalgebra $D$ (a concept due to Puig; see [17, Theorem 1.8]). This relationship suggests the following 'hyperfocal height zero' version of Brauer's height zero conjecture. Denote by $\operatorname{Irr}(D)$ a set of representatives of isomorphism classes of simple $K \otimes_{\mathcal{O}} D$-modules and by $\operatorname{Irr}_{0}(D)$ the subset corresponding to simple modules of $p^{\prime}$-degree. Let $\mathfrak{h y p}(\mathcal{F})$ denote the hyperfocal subgroup of $\mathcal{F}$. The focal subgroup $\mathfrak{f o c}(\mathcal{F})$ is the product $\mathfrak{h y p}(\mathcal{F}) P^{\prime}$ of the hyperfocal subgroup of $\mathcal{F}$ and the derived subgroup $P^{\prime}$ of $P$; see [1, Lemma 7.2].

Conjecture 1.2. With the notation above, assume that $K$ is a splitting field of $D$. Then, $\operatorname{Irr}(D)=\operatorname{Irr}_{0}(D)$ if and only if $\mathfrak{h y p}(\mathcal{F})$ is abelian.

Section 2 contains Clifford theoretic considerations regarding hyperfocal and source algebras. Theorem 1.1 is proved in Section 3. The last section contains some remarks around Conjecture 1.2 .

Remark 1.3. For $\chi \in \operatorname{Irr}(B)$ denote by $e_{\chi}$ the corresponding central primitive idempotent in $Z\left(K \otimes_{\mathcal{O}} B\right)$. If $e$ is an idempotent in $Z\left(K \otimes_{\mathcal{O}} B\right)$, then multiplication by $e$ induces a surjective $\mathcal{O}$-algebra homomorphism $B \rightarrow B e$; note that $B e$ is an $\mathcal{O}$-free quotient algebra of $B$ and an $\mathcal{O}$-subalgebra of $K \otimes_{\mathcal{O}} B$ but not necessarily a subalgebra of $B$. The integer $\sum_{\chi \in \operatorname{Irr}_{0}(B)} \chi(1)^{2}$ is equal to the $\mathcal{O}$-rank of the quotient algebra $B e$ of $B$, where $e=\sum_{\chi \in \operatorname{Irr}_{0}(B)} e_{\chi}$. If $B$ is nilpotent, then this algebra is Morita equivalent to the commutative symmetric algebra $\mathcal{O} P / P^{\prime}$, where $P^{\prime}$ is the derived subgroup of $P$. Indeed, through a Morita equivalence between $B$ and $\mathcal{O} P$, the height zero characters in $B$ correspond to the characters with degree one of $P$, and the intersection of the kernels of these is $P^{\prime}$. Okuyama and Tsushima proved in [14] that $B$ is Morita equivalent to a commutative (and necessarily symmetric) algebra if and only if $B$ is nilpotent with abelian defect groups. In a similar spirit one may ask whether there is a characterisation of blocks whose quotient $B e$ as defined above is Morita equivalent to a commutative symmetric $\mathcal{O}$-algebra.

Remark 1.4. If $|P: \mathfrak{f o c}(\mathcal{F})| \cdot|S: P|^{2}$ is the exact power of $p$ dividing $\sum_{\chi \in \operatorname{Irr}_{0}(B)} \chi(1)^{2}$, then the above theorem implies that $B$ is nilpotent, hence $\mathfrak{f o c}(\mathcal{F})$ is the derived subgroup $P^{\prime}$ of $P$. If $B$ is not nilpotent, then the highest power of $p$ dividing $\sum_{\chi \in \operatorname{Irr}_{0}(B)} \chi(1)^{2}$ is strictly bigger than $|P: \mathfrak{f o c}(P)| \cdot|S: P|^{2}$, and one might wonder whether it is always at least $\left|P: P^{\prime}\right| \cdot|S: P|^{2}$. This is, however, not the case. Let $p=3$. The group $H=A_{4} \times C_{4}$ has a faithful irreducible $\mathbb{F}_{3} H$-module $V$ of dimension 6 . Let $G=V H$ be the corresponding semidirect product. The group algebra $\mathcal{O} G$ has a unique block. If $P$ is a Sylow-3-subgroup of $G$, then $\left|P: P^{\prime}\right|=27$. This does not divide the sum of the squares of the $3^{\prime}$-degree characters, which is equal to $1548=2^{2} \cdot 3^{2} \cdot 43$.

\section{EXTENDING CHARACTERS OF THE HYPERFOCAL SUBALGEBRA}

Background material on focal and hyperfocal subgroups of fusion systems, as well as the fusion subsystem $O^{p}(\mathcal{F})$ of the fusion system $\mathcal{F}$ on $P$, can be found in [3. §7.5]. We refer to [17] for the notion and basic properties of hyperfocal subalgebras of source algebras of blocks. We show in this section that ordinary irreducible characters of the hyperfocal subalgebra of degree prime to $p$ extend to the source algebra in precisely $|P: \mathfrak{f o c}(\mathcal{F})|$ ways. The key ingredient is a special case of a result of Díaz, Glesser, Park and Stancu [4] which extends to fusion systems 
work of Gagola and Isaacs [6] on the transfer homomorphism. For a saturated fusion system $\mathcal{G}$ on a finite $p$-group $S$, we denote by $\tau_{S, \Omega}^{\mathcal{G}}: S \rightarrow S /[S, S]$ the transfer map with respect to some characteristic element $\Omega$ for $\mathcal{F}$ (see [4, Definition 2.5]), and by $T_{\mathcal{G}}$ the subgroup of $S$ containing $[S, S]$ such that $T_{\mathcal{G}} /[S, S]=\operatorname{Im}\left(\tau_{S, \Omega}^{\mathcal{G}}\right)$. By [4. Lemma 2.6], $T_{\mathcal{G}}$ is independent of the choice of $\Omega$. Further, if $U$ is a subgroup of $S$ containing $\mathfrak{h} \mathfrak{y} \mathfrak{p}(\mathcal{G})$, we denote by $\mathcal{G}_{U}$ the unique saturated subsystem of $\mathcal{G}$ on $U$ of $p$-power index (see [1, Theorem I.7.4]).

Proposition 2.1 ([4, Proposition 5.3]). Let $\mathcal{G}$ be a saturated fusion system on a finite p-group $S$ and let $U$ be a normal subgroup of $S$ containing $\mathfrak{h y p}(\mathcal{G})$. If $V$ is a subgroup of $U$ containing $T_{\mathcal{G}_{U}}[U, S]$, then $S / V \cong U / V \times T_{\mathcal{G}} V / V$.

Corollary 2.2. Let $\mathcal{G}$ be a saturated fusion system on a finite p-group $S$. Then $\mathfrak{h} \mathfrak{y} \mathfrak{p}(\mathcal{G}) /[\mathfrak{h} \mathfrak{y} \mathfrak{p}(\mathcal{G}), S]$ is a direct factor of $S /[\mathfrak{h y} \mathfrak{p}(\mathcal{G}), S]$.

Proof. Let $U=\mathfrak{h y p}(\mathcal{G})$. By the minimality of $\mathcal{G}_{U}$ as the normal saturated subsystem of $\mathcal{G}$ of $p$-power index, we have that $\mathfrak{f o c}\left(\mathcal{G}_{U}\right)=\mathfrak{h} \mathfrak{y p}\left(\mathcal{G}_{U}\right)=U$ (see [3, Theorem 7.53] and [1, Lemma I.7.2]). By Proposition 2.7 of [4] and the first line of its proof we have that

$$
U /[U, U] \cong \mathfrak{f o c}\left(\mathcal{G}_{U}\right) /[U, U] \times T_{\mathcal{G}_{U}} /[U, U] .
$$

Since $\mathfrak{f o c}\left(\mathcal{G}_{U}\right)=U$, it follows that $T_{\mathcal{G}_{U}}=[U, U]$. The corollary follows from Proposition 2.1 applied with $V=[U, S]$.

Keep the previous notation. In addition, set $Q=\mathfrak{h} \mathfrak{y} \mathfrak{p}(\mathcal{F})$ and $R=\mathfrak{f o c}(\mathcal{F})$, and set $A=i B i$, the source algebra of $B$ corresponding to the defect group $P$ of $B$ and the source idempotent $i$. By [17, Theorem 1.8] the hyperfocal subalgebra $D$ is the unique (up to $\left(A^{P}\right)^{\times}$-conjugation) $P$-stable unitary subalgebra of $A$ such that

$$
D \cap P i=Q i \text { and } A=D \otimes_{\mathcal{O} Q} \mathcal{O} P=\bigoplus_{u \in P / Q} D u \text {. }
$$

In particular, $D$ is a $Q$-interior $P$-algebra. We denote by $\hat{A}=K \otimes_{\mathcal{O}} A$ and $\hat{D}=$ $K \otimes_{\mathcal{O}} A$ the $K$-algebras obtained by extensions of scalars to $K$. The $K$-algebras $\hat{A}$ and $\hat{D}$ are semi-simple; indeed, for $\hat{A}$ this follows from the Morita equivalence between $A$ and $B$, and for $\hat{D}$ this is a consequence of the fact that $J(\hat{D}) \hat{A}$ is an ideal in $\hat{A}$, hence nilpotent, hence zero. By replacing $K$ by a suitable finite extension, we may and will assume that $\hat{A}$ and $\hat{D}$ are also split. We denote by $\operatorname{Irr}(A)$ (respectively $\operatorname{Irr}(D)$ ) a set of representatives of isomorphism classes of simple $\hat{A}$-modules (respectively $\hat{D}$-modules).

The $Q$-interior $P$-algebra structure of $D$ allows the usual notions of Clifford theory to be carried over to the inclusion of $\hat{D}$ in $\hat{A}$ (see [5]). In particular, $P$ acts on $\operatorname{Irr}(D)$. For $W \in \operatorname{Irr}(D)$ and $u \in P$ we denote by ${ }^{u} W \in \operatorname{Irr}(D)$ the image of $W$ under the action of $u$ and by $I_{P}(W)$ the stabiliser of $W$ in $P$. Note that $Q \leq I_{P}(W)$. We say that a simple $\hat{A}$-module $V$ covers $W$ if $W$ appears as a composition factor of $\operatorname{Res}_{\hat{D}}(V)$ and denote by $\operatorname{Irr}(A \mid W)$ the subset of $\operatorname{Irr}(A)$ which covers $W$. For $W \in \operatorname{Irr}(D)$ denote by $e_{W}$ the primitive central idempotent of $\hat{D}$ corresponding to $W$ and similarly for $\hat{A}$. Note that $\hat{D} e_{W} \cong \operatorname{Mat}_{\operatorname{dim}_{K}(W)}(K)$.

Suppose that $W \in \operatorname{Irr}(D)$ is $P$-stable. Then $\hat{D} e_{W} \cong \operatorname{Mat}_{\operatorname{dim}_{K}(W)}(K)$ is a $P$ algebra and by the Noether-Skolem theorem, every automorphism of $\hat{D} e_{W}$ is inner. Let $I$ be a set of coset representatives of $Q$ in $P$ containing 1. For each $y \in I$, choose 
an element $s_{y} \in\left(\hat{D} e_{W}\right)^{\times}$such that $s_{y} d\left(s_{y}\right)^{-1}={ }^{y} d$ for all $d \in \hat{D}$. For each $x \in Q$ and $y \in I$, set $s_{x y}=x s_{y}$. Then, for all $x, y \in P$, we have $s_{x} s_{y}\left(s_{x y}\right)^{-1} \in Z\left(\hat{D} e_{W}\right)^{\times}$. Identifying $Z\left(\hat{D} e_{W}\right)$ with $K$, the map $\beta: P \times P \rightarrow K^{\times}$given by $\beta\left(y, y^{\prime}\right)=$ $\left(s_{y y^{\prime}}\right)^{-1} s_{y} s_{y^{\prime}}$, where $y, y^{\prime} \in P$, is a 2-cocycle such that $\beta\left(x y, x^{\prime} y^{\prime}\right)=\beta\left(y, y^{\prime}\right)$ for all $x, x^{\prime} \in Q$. So, $\beta$ is the restriction to $P$ along the canonical map $P \rightarrow P / Q$ of a 2-cocycle $\bar{\beta}: P / Q \times P / Q \rightarrow K^{\times}$. We denote by $\alpha_{W}$ the image of $\bar{\beta}$ in $H^{2}\left(P / Q, K^{\times}\right)$. Then $\alpha_{W}$ is independent of the choice of $I$ and the choice of elements $s_{y}, y \in I$.

For $L$ a finite group and $\alpha \in H^{2}\left(L, K^{\times}\right)$, we denote by $K_{\alpha} L$ a twisted group algebra of $L$ over $K$ corresponding to $\alpha$. The following proposition is a rewording of some of the results in [5].

Proposition 2.3. Let $V \in \operatorname{Irr}(A)$ and $W \in \operatorname{Irr}(D)$.

(i) $\operatorname{Res}_{\hat{D}}(V)$ is semi-simple, and if $V$ covers $W$, then

$$
\operatorname{Res}_{\hat{D}}(V) \cong m_{V} \sum_{u \in P / I_{P}(W)}{ }^{u} W .
$$

(ii) $V$ covers $W$ if and only if $e_{W} e_{V} \neq 0$.

(iii) Suppose that $W \in \operatorname{Irr}(A)$ is P-stable. Then

$$
\hat{A} e_{W} \cong \hat{D} e_{W} \otimes_{K} K_{\alpha_{W}-1}(P / Q)
$$

as $K$-algebras. In particular, there is a bijection $V \rightarrow V_{0}$ between $\operatorname{Irr}(A \mid W)$ and $\operatorname{Irr}\left(K_{\alpha_{W}}(P / Q)\right)$ such that $\operatorname{dim}_{K}(V)=\operatorname{dim}_{K}(W) \operatorname{dim}_{K}\left(V_{0}\right)$.

(iv) Suppose that $V$ covers $W$. If $\operatorname{dim}_{K}(V)$ is prime to $p$, then $W$ is P-stable, $\operatorname{dim}_{K}(W)=\operatorname{dim}_{K}(V)$ is prime to $p$ and $\alpha_{W}$ is trivial.

(v) Suppose that $W$ is $P$-stable, $\operatorname{dim}_{K}(W)$ is prime to $p$, and $\alpha_{W}$ is trivial. Then there are $[P: R]$ elements in $\operatorname{Irr}(A \mid W)$ whose dimension is prime to $p$ and they all have the same degree $\operatorname{dim}_{K}(W)$.

Proof. See the proof of [5, Theorem 1.6].

Corollary 2.2 provides the following key extendibility result.

Proposition 2.4. Suppose that $W \in \operatorname{Irr}(D)$ is $P$-stable and that $\operatorname{dim}_{K}(W)$ is prime to $p$. Then $\alpha_{W}$ is trivial. Equivalently, $W$ extends to $|P: R|$ pairwise nonisomorphic simple $\hat{A}$-modules.

Proof. Let $\delta: Q \rightarrow K^{\times}$be defined by $\delta(x)=\operatorname{det}\left(x e_{W}\right)$, where $x \in Q$. Then $\delta$ is a $P$-stable group homomorphism. Since every element of $P$ acts as an inner automorphism of $\hat{D} e_{W},[Q, P] \leq \operatorname{Ker}(\delta)$. Thus, by Corollary 2.2, $\delta$ extends to a group homomorphism from $P$ to $K^{\times}$. Choose such an extension, and by abuse of notation, denote it again by $\delta$. Let $I$ be a set of coset representatives of $Q$ in $P$. For each $y \in I$, choose the element $s_{y}$ in $\left(\hat{D} e_{W}\right)^{\times}$as in the definition of $\alpha_{W}$ such that $\operatorname{det}\left(s_{y}\right)=\delta(y)$. This is possible as the dimension of $W$ is prime to $p$ and $\delta(y)$ is a $|P|$-th root of unity (and since we may assume that $K$ contains a $|P|$-th root of unity). Then for all $x \in P$, $\operatorname{det}\left(s_{x}\right)=\delta(x)$ and consequently, $\operatorname{det}\left(s_{x y}\right)=$ $\operatorname{det}\left(s_{x}\right) \operatorname{det}\left(s_{y}\right)$ for all $x, y \in P$. By the definition of $\beta$, we have $s_{x} s_{y}=\beta(x, y) s_{x y}$ for all $x, y \in P$. Hence, for all $x, y \in P$, we have $\beta(x, y)^{n}=1$, where $n=\operatorname{dim}_{K}(W)$. Consequently, $\alpha_{W} \in H^{2}\left(P / Q, K^{\times}\right)$has order dividing $n$. Since $n$ is prime to $p$ and $H^{2}\left(P / Q, K^{\times}\right)$is a $p$-group, it follows that $\alpha_{W}=1$, as claimed. 


\section{Proof of Theorem 1.1}

Proof of Theorem 1.1. As before, we denote by $A=i B i$ the source algebra of $B$ with respect to the defect group $P$ and the source idempotent $i \in B^{P}$. We denote by $\mathcal{F}$ the fusion system of $B$ on $P$ determined by $i$. We set $R=\mathfrak{f o c}(\mathcal{F})$ and $Q=$ $\mathfrak{h} \mathfrak{y} \mathfrak{p}(\mathcal{F})$. We first prove the equivalence of (i) and (ii). Recall from [17, Introduction] or [18] that the Broué-Puig *-construction from [2] on characters yields a free action of $P / R$ on $\operatorname{Irr}_{0}(B)$. Let $\chi, \chi^{\prime} \in \operatorname{Irr}_{0}(B)$. If $\chi, \chi^{\prime}$ lie in the same orbit of $P / R$, then from the definition of the $*$-construction, it is immediate that $\chi(x)=\chi^{\prime}(x)$ for all $p$-regular elements of $G$, hence $\chi(j)=\chi^{\prime}(j)$ for any idempotent $j$ of $\mathcal{O} G$ (as $\chi$ and $\chi^{\prime}$ have the same decomposition numbers). In particular, if $\chi$ and $\chi^{\prime}$ are in the same orbit of $P / R$ on $\operatorname{Irr}_{0}(B)$, then $\chi(1)=\chi^{\prime}(1)$ and $\chi(i)=\chi^{\prime}(i)$. Thus, letting $\mathcal{S}$ denote a set of representatives of the $P / R$-orbits on $\operatorname{Irr}_{0}(B)$, we have that

$$
\begin{aligned}
\sum_{\chi \in \operatorname{Irr}_{0}(B)} \chi(1)^{2} & =|P: R| \sum_{\chi \in \mathcal{S}} \chi(1)^{2} ; \\
\sum_{\chi \in \operatorname{Irr}_{0}(B)} \chi(i)^{2} & =|P: R| \sum_{\chi \in \mathcal{S}} \chi(i)^{2} .
\end{aligned}
$$

Since $|S: P|$ divides $\chi(1)$ for all $\chi \in \operatorname{Irr}(B)$, it follows from the above that in order to prove the equivalence of (i) and (ii), it suffices to prove that the integer $\sum_{\chi \in \mathcal{S}} \chi(i)^{2}$ belongs to $J(\mathcal{O})$ if and only if the integer $\sum_{\chi \in \mathcal{S}}\left(\frac{\chi(1)}{|S: P|}\right)^{2}$ belongs to $J(\mathcal{O})$.

Let $\omega_{B}: Z(B) \rightarrow Z(B) / J(Z(B))=k$ be the canonical surjection. By [15] or [19, 9.3], $\operatorname{Tr}_{P}^{G}(i)$ is an invertible element of $Z(B)$, hence $\omega_{B}\left(\operatorname{Tr}_{P}^{G}(i)\right) \neq 0$. Recall that for any $z \in Z(B)$, and any $\chi \in \operatorname{Irr}(B)$, we have $\frac{\chi(z)}{\chi(1)} \in \mathcal{O}$ and $\omega_{B}(z)=\frac{\overline{\chi(z)}}{\chi(1)}$, where for $x \in \mathcal{O}$ we denote by $\bar{x}$ the image in $k$ of $x$ under the canonical surjection $\mathcal{O} \rightarrow k$. Thus, for any $\chi \in \operatorname{Irr}(B)$, we have

$$
0 \neq \omega_{B}\left(\operatorname{Tr}_{P}^{G}(i)\right)=\overline{\frac{\chi\left(\operatorname{Tr}_{P}^{G}(i)\right)}{\chi(1)}}=\overline{|G: P| \frac{\chi(i)}{\chi(1)}} .
$$

Choose $\lambda \in \mathcal{O}^{\times}$with $\bar{\lambda}=\omega_{B}\left(\operatorname{Tr}_{P}^{G}(i)\right)$. The above shows that for any $\chi \in \operatorname{Irr}(B)$, there exists an element $t_{\chi} \in J(\mathcal{O})$ such that

$$
|G: P| \frac{\chi(i)}{\chi(1)}=\lambda+t_{\chi} .
$$

Since $|G: S|$ is invertible in $\mathcal{O}$, by suitably replacing $\lambda$ and $t_{\chi}$, we obtain that there exists $\lambda \in \mathcal{O}^{\times}$such that for all $\chi \in \operatorname{Irr}(B)$, there exists $t_{\chi} \in J(\mathcal{O})$ such that

$$
|S: P| \frac{\chi(i)}{\chi(1)}=\lambda+t_{\chi}
$$

Since $t_{\chi} \in J(\mathcal{O})$, we have that

$$
\sum_{\chi \in \mathcal{S}} \chi(i)^{2}=\sum_{\chi \in \mathcal{S}}\left(\lambda+t_{\chi}\right)^{2}\left(\frac{\chi(1)}{|S: P|}\right)^{2} \equiv \lambda^{2} \sum_{\chi \in \mathcal{S}}\left(\frac{\chi(1)}{|S: P|}\right)^{2}(\bmod J(\mathcal{O})) .
$$

The equivalence of (i) and (ii) follows. If (iii) holds, then by the above, the height zero characters in $B$ form a unique regular $P / R$-orbit with respect to the *-construction; in particular, all height zero characters of $B$ have the same degree 
with $p$-part $|S: P|$, and hence (iii) implies (i). We prove next that (ii) implies (iv). The strategy is the same as used for [9, Lemma 4]. Let $\mathcal{V}$ denote the subset of $\operatorname{Irr}_{0}(D)$ consisting of $P$-stable elements. Note that $\chi \in \operatorname{Irr}_{0}(B)$ is of height zero if and only if $\chi(i)$ is prime to $p$. Moreover, $\chi(i)$ is the dimension of the simple $\hat{A}$-module corresponding to $\chi$ under the canonical Morita equivalence between $B$ and $A$. By Proposition 2.3 (i)-(iv) and Proposition 2.4 it follows that

$$
\sum_{\chi \in \operatorname{Irr}_{0}(B)} \chi(i)^{2}=|P: R| \sum_{W \in \mathcal{V}} \operatorname{dim}_{K}(W)^{2} .
$$

In particular, $|P: R|$ divides $\sum_{\chi \in \operatorname{Irr}_{0}(B)} \chi(i)^{2}$ and we have

$$
\frac{1}{|P: R|} \sum_{\chi \in \operatorname{Irr}_{0}(B)} \chi(i)^{2}=\sum_{W \in \mathcal{V}} \operatorname{dim}_{K}(W)^{2} .
$$

If $W^{\prime} \in \operatorname{Irr}(D) \backslash \mathcal{V}$, then either $p$ divides the dimension of $W^{\prime}$ or the size of the $P$-orbit of $W^{\prime}$ is divisible by $p$. Hence,

$$
\sum_{W \in \mathcal{V}} \operatorname{dim}_{K}(W)^{2} \equiv \sum_{W \in \operatorname{Irr}(D)} \operatorname{dim}_{K}(W)^{2}(\bmod p) .
$$

Assume that $\frac{1}{|P: R|} \sum_{\chi \in \operatorname{Irr}_{0}(B)} \chi(i)^{2}$ is a $p^{\prime}$-integer. Then by the above, $\operatorname{dim}_{K}(\hat{D})=$ $\sum_{W \in \operatorname{Irr}(D)} \operatorname{dim}_{K}(W)^{2}$ is a $p^{\prime}$-integer. The hyperfocal subalgebra $D$ is a direct summand of $A$ as a left $D$-module, hence projective as a left $\mathcal{O} Q$-module. In particular, $|Q|$ divides the $\mathcal{O}$-rank of $D$. Thus $Q=1$, which means that $\mathcal{F}$ and hence $B$ are nilpotent. This proves that (ii) implies (iv). As mentioned earlier, the fact that (iv) implies any of (i), (ii), (iii) is immediate from the structure theorem [16, Theorem 1.6] for source algebras of nilpotent blocks.

\section{Around Conjecture 1.2}

As before, $G$ is a finite group, $B$ a block algebra of $\mathcal{O} G$ with corresponding block idempotent $b=1_{B}$, and $P$ a defect group of $B$. Let $i \in B^{P}$ be a source idempotent and $A=i B i$ the corresponding source algebra.

Proposition 4.1. Suppose $P$ is abelian. Then $\operatorname{Irr}_{0}(D)=\operatorname{Irr}(D)$.

Proof. This follows from the forward direction of the height zero conjecture (proved by Kessar and Malle in [10]), and Proposition 2.3.

Proposition 4.2. Conjecture 1.2 holds for blocks with a normal defect group.

Proof. Suppose that the defect group $P$ of $B$ is normal in $G$. By Külshammer's structure theorem in [12, the source algebra $A$ is isomorphic to a twisted group algebra of $P \rtimes E$, where $E$ is the inertial quotient of $B$. From this it follows that a hyperfocal subalgebra is isomorphic to a twisted group algebra of $Q \rtimes E$. The result follows.

The block $B$ is said to be of principal type if for any subgroup $Q$ of $P$, the idempotent $\operatorname{Br}_{Q}(b)$ is a block of $k C_{G}(Q)$, or equivalently, if the algebra $B(Q)=$ $k C_{G}(Q) \mathrm{Br}_{Q}(b)$ is a block algebra of $k C_{G}(Q)$. In that case, we have $e_{P}=\operatorname{Br}_{P}(b)$, there is a unique local point $\gamma$ of $P$ on $B$, and the fusion system $\mathcal{F}$ of $B$ on $P$ is equal to the fusion system $\mathcal{F}_{P}(G)$ induced by conjugation of elements in $G$ on subgroups of $P$. The principal block of $\mathcal{O} G$ is of principal type by Brauer's third 
main theorem. The following two observations are well-known and included for convenience; the proofs are routine block theory. Recall that the multiplicity $m_{\gamma}$ of a local point of $P$ on $B$ is the number $|\gamma \cap I|$ of elements of $\gamma$ which appear in a primitive orthogonal idempotent decomposition $I$ of $b$ in $B^{P}$.

Lemma 4.3. Suppose that the defect group $P$ of $B$ is a Sylow-p-subgroup of $G$. Let $\gamma$ be a local point of $P$ on $\mathcal{O} G^{P}$. Then the multiplicity $m_{\gamma}$ of $\gamma$ on $B$ is prime to $p$ and divides the order of $C_{G}(P)$.

Proof. Let $e$ be the unique block of $k C_{G}(P)$ such that $\operatorname{Br}_{P}(\gamma) \subseteq k C_{G}(P) e$. Then $\operatorname{Br}_{P}(\gamma)$ is the unique point of $k C_{G}(P) e$. Thus $m_{\gamma}$ is the dimension of the unique (up to isomorphism) simple $k C_{G}(P) e$-module $V$. Since $P$ is a Sylow- $p$-subgroup of $G$, it follows that $C_{G}(P)=Z(P) \times C^{\prime}$ for some $p^{\prime}$-subgroup $C^{\prime}$ of $C_{G}(P)$, and hence $e=e_{\eta}$, where $\eta$ is an irreducible character of $C^{\prime}$ with coefficients in $k$ and $e_{\eta}$ is the corresponding central primitive idempotent in $k C^{\prime}$ (which makes sense as $\left|C^{\prime}\right|$ is prime to $p$ ). Thus $\operatorname{dim}_{k}(V)=\eta(1)$ divides $\left|C^{\prime}\right|$, whence the result.

Proposition 4.4. Suppose that $B$ is of principal type and that $P$ is a Sylow-psubgroup of $G$. Set $N=O^{p}(G)$. Then $b$ is a block idempotent of $\mathcal{O} N$. Setting $C=$ $\mathcal{O N b}$, the following hold.

(i) The block $C$ of $\mathcal{O} N$ is of principal type, with a defect group $Q=P \cap N=$ $\mathfrak{h} \mathfrak{n p}(\mathcal{F})$ and fusion system $O^{p}(\mathcal{F})$.

(ii) The algebra $C^{P}$ contains a $\left(B^{P}\right)^{\times}$-conjugate of $i$.

(iii) If $i \in C^{P}$, then $D=i C i$ is a hyperfocal subalgebra of the source algebra $A=i B i$ of $B$.

(iv) If $i \in C^{P}$, then there is a source idempotent $j \in(i C i)^{Q}$ of the block $C$ belonging to the unique local point $\delta$ of $Q$ on $C$. The multiplicity $m_{\delta}^{\gamma}$ of $\delta$ on $i C i$ is prime to $p$, and for any $\chi \in \operatorname{Irr}(C)$ we have $\chi(i) \equiv m_{\delta}^{\gamma} \chi(j) \bmod p$.

(v) All irreducible characters of $i C i$ have degree prime to $p$ if and only if all irreducible characters of $j C j$ have degree prime to $p$.

Proof. Since a block idempotent is supported on $p^{\prime}$-elements, we have $b \in(\mathcal{O} N)^{G}$. Thus $b$ is a $G$-conjugacy class sum of blocks of $\mathcal{O} N$. As $G=N P$, the idempotent $b$ is in fact a $P$-conjugacy class sum of blocks of $\mathcal{O} N$. Since $\operatorname{Br}_{P}(b) \neq 0$, this implies that $b$ is a block of $\mathcal{O} N$. Then $Q=P \cap N$ is a Sylow- $p$-subgroup of $N$, hence a defect group of the block algebra $C=\mathcal{O} N b$ of $\mathcal{O} N$. By Puig's hyperfocal subgroup theorem, we have $Q=\mathfrak{h y p}(\mathcal{F})$. Let $R$ be a subgroup of $Q$. We need to show that $f=\operatorname{Br}_{R}(b)$ is a block of $k C_{N}(R)$. After replacing $R$ by a conjugate, if necessary, we may assume that $C_{P}(R)$ is a Sylow-p-subgroup of $C_{G}(R)$. Since $C_{N}(R)$ is normal of $p$-power index in $C_{G}(R)$, it follows that $C_{G}(R)=C_{N}(R) C_{P}(R)$. We have $\operatorname{Br}_{C_{P}(R)}(f) \neq 0$ because $f$ is a block of $k C_{G}(R)$ with defect group $C_{P}(R)$. Arguing as above for $b$ it follows that $f$ is a $C_{P}(R)$-conjugacy class of block idempotents of $k C_{N}(R)$, and hence the condition $\operatorname{Br}_{C_{P}(R)}(f) \neq 0$ implies that $f$ remains a block idempotent of $k C_{N}(R)$. Thus the block $C$ is of principal type, and hence $C$ has the fusion system $\mathcal{F}_{Q}(N)$, which is equal to $O^{p}(\mathcal{F})$. This proves (i).

Statement (ii) follows as the inclusion $C \rightarrow B$ is a semi-covering (cf. 13, 3.9, $3.16]$ ) and therefore any primitive (local) idempotent in $C^{P}$ remains primitive (local) in $B^{P}$. Thus the unique local point $\gamma$ of $P$ on $B$ contains an element in $C$. Choosing such an $i \in \gamma \cap C$, it follows that $i C i$ is a unitary $P$-stable subalgebra of $i B i$ such that $i C i \cap P=i(P \cap N) i=Q i$. 
Statement (iii) follows by the uniqueness of hyperfocal subalgebras. By [17, 4.2], the group $Q$ has a unique local point $\delta$ on $D=i C i$. This is then necessarily a defect pointed group of $C$, and hence any $j \in \delta$ is a source idempotent of $C$. If $j^{\prime}$ belongs to a nonlocal point of a subgroup $R$ of $P$ on $\mathcal{O} G$, then $j^{\prime}$ can be written as a trace from a proper subgroup of $R$, and hence $\chi\left(j^{\prime}\right) \in J(\mathcal{O})$ for any $\chi \in \operatorname{Irr}(G)$. Thus, if $\chi \in \operatorname{Irr}(B)$, then $\chi(1) \equiv m_{\gamma} \chi(i) \bmod J(\mathcal{O})$, since $\gamma$ is the unique local point of $P$ on $B$. Similarly, we have $\chi(i) \equiv m_{\delta}^{\gamma} \chi(j) \bmod J(\mathcal{O})$. Since $P$ is a Sylow- $p$-subgroup of $G$, it follows that the height zero characters in $B$ are the characters of degree prime to $p$ in $B$. Thus the previous lemma and the above congruence applied to a height zero character yield together that $m_{\delta}^{\gamma}$ is prime to $p$. This proves (iv).

Multiplication by $j$ induces a Morita equivalence between the hyperfocal subalgebra $i C i$ of $B$ and the source algebra $j C j$ of $C$. In particular, multiplication by $j$ induces a bijection between irreducible characters of $i C i$ and $j C j$. Thus (v) follows from (iv).

Proposition 4.5. Conjecture 1.2 holds for blocks of finite p-solvable groups.

Proof. Suppose that $G$ is $p$-solvable. Then $B$ is source algebra equivalent to a block of principal type of a subgroup $H$ of $G$ such that $P$ is a Sylow-p-subgroup of $H$ and such that the block idempotent belongs to $\mathcal{O} O_{p^{\prime}} H$; this is a version of FongReynolds reduction, as presented, for instance, in [8, Theorem 5.1]. Thus we may assume that $B$ is of principal type and that $P$ is a Sylow- $p$-subgroup of $G$. Set $N=O^{p}(G)$. By 4.4, the block idempotent $b$ of $B$ remains a block idempotent in $\mathcal{O} N$, and the group $Q=N \cap P=\mathfrak{h y p}(\mathcal{F})$ is a defect group of $C=\mathcal{O} N b$. It follows from Proposition 4.4 (v) that all irreducible characters of a hyperfocal subalgebra $D$ of $B$ have degree prime to $p$ if and only if all irreducible characters in $C$ have degree prime to $p$. Since by a result of Gluck and Wolf [7] the height zero conjecture holds for blocks of $p$-solvable groups, it follows that this is equivalent to $Q$ being abelian.

Since [10] and [7] invoke the classification of finite simple groups, so do the above proofs of Conjecture 1.2 for blocks with an abelian defect group or blocks of $p$-solvable finite groups.

Proposition 4.6. For blocks of principal type and maximal defect, Brauer's height zero conjecture implies Conjecture 1.2 .

Proof. Suppose that Brauer's height zero conjecture holds for blocks of principal type and maximal defect. Suppose that $B$ is of principal type and that its defect group is a Sylow-p-subgroup of $G$. Let $N, C, i, j$ be as in Proposition 4.4. By Proposition 4.4 (v), all irreducible characters of the hyperfocal algebra $D=i C i$ have degree prime to $p$ if and only if all irreducible characters of the source algebra $j C j$ of $C$ have degree prime to $p$, which by Brauer's height zero conjecture (assumed to be true) applied to the block $C$ is true if and only if $Q$ is abelian.

Remark 4.7. The converse implication in Proposition 4.6 holds for the 'forward' direction of the two conjectures. More precisely, suppose that Conjecture 1.2 holds for all blocks of principal type and maximal defect. Suppose that $B$ is of principal type and that $P$ is a Sylow- $p$-subgroup of $G$. Let $N, C, i, j$ be as in Proposition 4.4. It follows from Proposition 2.3 that all irreducible characters of $i B i$ have $p^{\prime}$-degree if and only if all irreducible characters of $i C i$ are $P$-stable, have $p^{\prime}$-degree, $P / Q$ is abelian, and all 2-cocycles $\alpha_{W}$ as in Proposition 2.3 are trivial. Since we assume 
Conjecture 1.2 to be true, this is equivalent to requiring that all irreducible characters of $i C i$ are $P$-stable, the groups $Q, P / Q$ are both abelian, and all $\alpha_{W}$ as before are trivial. We do not know whether these conditions force $P$ to be abelian. We can show that these conditions are satisfied if $P$ is abelian. Indeed, if $P$ is abelian, then $Q$ and $P / Q$ are trivially abelian. By a theorem of Knörr in [11, every $\mathcal{O}$-free $B$-module affording an irreducible character $\chi$ has vertex $P$. But then any character of $C$ covered by $\chi$ must be $P$-stable (because otherwise one could write $\chi$ as being induced from a proper subgroup of $G$ containing $N$, hence not containing $P$, yielding a lattice with vertex smaller than $P$ ). All characters of $i C i$ have $p^{\prime}$-degree, so all $\alpha_{W}$ are trivial by Proposition 2.4.

\section{REFERENCES}

[1] Michael Aschbacher, Radha Kessar, and Bob Oliver, Fusion systems in algebra and topology, London Mathematical Society Lecture Note Series, vol. 391, Cambridge University Press, Cambridge, 2011. MR2848834 (2012m:20015)

[2] Michel Broué and Lluís Puig, Characters and local structure in G-algebras, J. Algebra 63 (1980), no. 2, 306-317, DOI 10.1016/0021-8693(80)90074-5. MR570714 (81j:20021)

[3] David A. Craven, The theory of fusion systems, Cambridge Studies in Advanced Mathematics, vol. 131, Cambridge University Press, Cambridge, 2011. An algebraic approach. MR2808319 (2012f:20028)

[4] Antonio Díaz, Adam Glesser, Sejong Park, and Radu Stancu, Tate's and Yoshida's theorems on control of transfer for fusion systems, J. Lond. Math. Soc. (2) 84 (2011), no. 2, 475-494, DOI 10.1112/jlms/jdr019. MR2835340 (2012k:20038)

[5] Yun Fan, Hyperfocal subalgebras of blocks and computation of characters, J. Algebra 322 (2009), no. 10, 3681-3692, DOI 10.1016/j.jalgebra.2009.05.045. MR2568357 (2011c:20012)

[6] Stephen M. Gagola Jr. and I. M. Isaacs, Transfer and Tate's theorem, Arch. Math. (Basel) 91 (2008), no. 4, 300-306, DOI 10.1007/s00013-008-2910-7. MR2447543 (2009g:20031)

[7] David Gluck and Thomas R. Wolf, Brauer's height conjecture for p-solvable groups, Trans. Amer. Math. Soc. 282 (1984), no. 1, 137-152, DOI 10.2307/1999582. MR728707 (85i:20014)

[8] M. E. Harris and M. Linckelmann, On the Glauberman and Watanabe correspondences for blocks of finite p-solvable groups, Trans. Amer. Math. Soc. 354 (2002), no. 9, 3435-3453 (electronic), DOI 10.1090/S0002-9947-02-02990-2. MR1911507(2003c:20008)

[9] I. M. Isaacs, Recovering information about a group from its complex group algebra, Arch. Math. (Basel) 47 (1986), no. 4, 293-295, DOI 10.1007/BF01191352. MR866514 (87m:20017)

[10] Radha Kessar and Gunter Malle, Quasi-isolated blocks and Brauer's height zero conjecture, Ann. of Math. (2) 178 (2013), no. 1, 321-384, DOI 10.4007/annals.2013.178.1.6. MR3043583

[11] Reinhard Knörr, On the vertices of irreducible modules, Ann. of Math. (2) 110 (1979), no. 3, 487-499, DOI 10.2307/1971234. MR554380 (81f:20013)

[12] Burkhard Külshammer, Crossed products and blocks with normal defect groups, Comm. Algebra 13 (1985), no. 1, 147-168, DOI 10.1080/00927878508823154. MR768092 (86c:20015)

[13] Burkhard Külshammer and Lluís Puig, Extensions of nilpotent blocks, Invent. Math. 102 (1990), no. 1, 17-71, DOI 10.1007/BF01233419. MR1069239(91i:20009)

[14] Tetsuro Okuyama and Yukio Tsushima, Local properties of p-block algebras of finite groups, Osaka J. Math. 20 (1983), no. 1, 33-41. MR695615 (84i:20012)

[15] Claudine Picaronny and Lluís Puig, Quelques remarques sur un thème de Knörr (French), J. Algebra 109 (1987), no. 1, 69-73, DOI 10.1016/0021-8693(87)90164-5. MR898337 (88e:20013)

[16] Lluís Puig, Nilpotent blocks and their source algebras, Invent. Math. 93 (1988), no. 1, 77-116, DOI 10.1007/BF01393688. MR943924 (89e:20023)

[17] Lluis Puig, The hyperfocal subalgebra of a block, Invent. Math. 141 (2000), no. 2, 365-397, DOI 10.1007/s002220000072. MR1775217 (2001h:20012)

[18] Geoffrey R. Robinson, On the focal defect group of a block, characters of height zero, and lower defect group multiplicities, J. Algebra 320 (2008), no. 6, 2624-2628, DOI 10.1016/j.jalgebra.2008.04.032. MR 2441776(2009f:20010) 
[19] Jacques Thévenaz, Duality in G-algebras, Math. Z. 200 (1988), no. 1, 47-85, DOI 10.1007/BF01161746. MR972396 (90b:20009)

Department of Mathematics, City University, London EC1V 0HB, Great Britain

E-mail address: radha.kessar.1@city.ac.uk

Department of Mathematics, City University, London EC1V 0HB, Great Britain

E-mail address: markus.linckelmann.1@city.ac.uk SPAIN

Departament D’Àlgebra, Universitat de València, Dr. Moliner 50, 46100 Burjassot,

E-mail address: gabriel.navarro@uv.es 